\title{
Study of Microbiological Profile and Antibiotic Susceptibility of Blood Stream Infections in Tertiary Care Hospital
}

\author{
K. Vidyasagar* and D. Venkatesha
}

Department of Microbiology, Adichunchanagiri Institute of Medical Sciences, BG Nagara, Nagamangala (Taluk), Mandya (Dist)-PIN: 571448, Karnataka, India

*Corresponding author

\section{A B S T R A C T}

\begin{tabular}{|c|}
\hline Keywords \\
\hline $\begin{array}{l}\text { Blood stream } \\
\text { infections (BSI), } \\
\text { Microbiological } \\
\text { profile, Antimicrobial } \\
\text { susceptibility }\end{array}$ \\
\hline Article Info \\
\hline $\begin{array}{l}\text { Accepted: } \\
10 \text { June } 2019 \\
\text { Available Online: } \\
10 \text { July } 2019\end{array}$ \\
\hline
\end{tabular}

Blood stream infections (BSIs) can lead to life-threatening sepsis and are globally associated with high morbidity and mortality. Early diagnosis plays a crucial role in managing BSI. Objective is to identify the pathogens causing blood stream infections and to know their antibiotic sensitivity pattern. This was a retrospective study of 1 year duration. A total of 1332 blood samples from clinically diagnosed cases of blood stream infections received in the microbiology laboratory were included in the study. Blood samples were processed and isolates were identified by standard biochemical tests and antibiotic susceptibility testing was done by Kirby Bauer disc diffusion methods as per CLSI guidelines. Out of 1332 blood samples received, 204 (15.3\%) samples showed growth and $1128(84.68 \%)$ samples showed no growth, with total percentage of culture positivity being $15.3 \%$. Among 204 positive cultures, 202 (99\%) showed bacterial growth and $2(0.98 \%)$ were Candida spp. Bacteremia due to Grampositive pathogens was more common compared to Gram-negative pathogens. The present study provides information about pathogens responsible for blood stream infections and their antibiotic susceptibility. Antibiotic susceptibility pattern of isolates provides useful guidelines to clinicians in initiating empiric therapy and help in management of blood stream infections.

\section{Introduction}

Blood stream infections (BSIs) can lead to life-threatening sepsis and are globally associated with high morbidity and mortality. ${ }^{[1]}$ Blood stream infections (BSI) are defined by the presence and active multiplication of microorganisms in the blood stream. ${ }^{[2]}$ BSI by the place of acquisition is categorized either community associated or hospital associated. Blood stream infections occur when bacteria enter the blood stream from either a primary focus of infection in an organ (UTI, Pneumonia, meningitis...), a wound or via an indwelling or implanted device. ${ }^{[3]}$ Health care associated (HCA) BSIs can occur as complications following medical and surgical procedures or the insertion of an intravascular or indwelling device. ${ }^{[4]}$ Blood stream infections (BSIs) have serious 
consequences such as shock, disseminated intravascular coagulation, multiple organ failure, and even death. Early diagnosis plays a crucial role in managing BSI, and hence, prompt detection of such infections is a critical function of clinical microbiology laboratories. ${ }^{[5]}$

BSI can be caused by both Gram-positive and Gram-negative microorganisms as well as fungi. Common Gram negative bacteria are Escherichia coli, Klebsiella spp, Enterobacter spp, Proteus spp, Salmonella typhi, Pseudomonas aeruginosa, Acinetobacter spp, Haemophilus influenza, Brucella spp and Neisseria meningitidis. Common Gram positive bacteria are Staphylococcus aureus, Coagulase negative Staphylococci (CONS), Enterococci and alpha haemolytic (viridans) streptococci. ${ }^{[6]}$

Blood culture is the gold standard for the detection of blood stream infection. ${ }^{[7]}$ One of the main complication in the treatment of BSI is the increasing resistance of bacteria to antibiotics.

Emerging drug resistance among blood stream pathogens limit therapeutic options and complicate patient's management. ${ }^{[8]}$ Today the only way to reduce mortality due to blood stream infection is early diagnosis and appropriate antimicrobial therapy at the earliest.

The aim of the present study to identify the pathogens causing blood stream infection and to know their antibiotic sensitivity pattern, thus providing useful guidance to clinicians to antibiotic therapy.

\section{Materials and Methods}

This was a retrospective study conducted for a period of one year in a tertiary care hospital. A total of 1332 blood samples from clinically diagnosed cases of blood stream infections received in the microbiology laboratory were included in the study.

Blood samples were collected from clinically suspected bacteremia cases before the administration of antibiotics under aseptic precautions and inoculated into brain heart infusion broth. A volume of 5-10 $\mathrm{ml}$ from adults and $2-3 \mathrm{ml}$ from pediatric patients were obtained for culture. The culture bottles were incubated at $37^{\circ} \mathrm{C}$ aerobically for $18-24$ hours and periodic subcultures were done onto MacConkey agar and blood agar on day 2, day 4 and finally on day 7 and samples were reported as no growth after 7 days of aerobic incubation. Bacterial growth on the subcultures was identified by colony morphology, Gram staining, and standard biochemical tests. ${ }^{[6]}$ Antibiotic susceptibility testing was done by Kirby Bauer disc diffusion methods as per CLSI guidelines. Cefoxitin disc diffusion method used to identify Methicillin resistant Staphylococcus aureus (MRSA) and Methicillin resistant Coagulase negative Staphylococci (MRCONS) among Staphylococcus aureus and Coagulase negative Staphylococci respectively. ${ }^{[9]}$ Extended spectrum betalactamases (ESBL) in Gram-negative bacilli were studied by phenotypic method with ceftazidime $(30 \mu \mathrm{g})$ and ceftazidime + clavulanic acid $(30 \mu \mathrm{g}+10 \mu \mathrm{g})$ as per CLSI guidelines. ${ }^{[9]}$ MDR (Multi drug resistant) was defined as non-susceptibility to at least one agent in three or more antimicrobial categories. ${ }^{[9]}$

\section{Results and Discussion}

In the present study, 1332 blood samples were received and processed for aerobic culture. Out of 1332 blood samples, 204 (15.3\%) samples showed growth, 1128(84.68\%) samples showed no growth and with total percentage of culture positivity being $15.3 \%$. 
Out of 1332 blood samples received, 793 $(59.53 \%)$ were from male and 539(40.46\%) were female, maximum from the age group of <1year (37.31\%), followed by $1-10$ year $(19.29 \%), 21-30 y e a r s \quad(7.73 \%)$ and $31-40$ years $(7.28 \%$ ) respectively (Figure 1$)$.

The ward wise distribution of samples includes 1254(94.19\%) received from IPD, $72(5.40 \%)$ from ICU and $6(0.45 \%)$ from OPD.

Among 204 positive cultures, 202(99\%) showed bacterial growth and 2(0.98\%) were Candida spp. Out of 202 (99\%) bacterial growth, Gram positive organisms were $128(62.74 \%)$ and Gram negative organisms were $74(36.27 \%$ ) respectively (Table 1$)$.

Among Gram positive isolates (128), the most predominant isolate was Coagulase negative Staphylococci (CONS) 114(89\%) followed by Staphylococcus aureus 8(6.25\%) and Enterococcus faecalis 6(4.68\%) (Table 2).

Methicillin resistance Coagulase negative Staphylococci (MRCONS) was found in $82.45 \%$ of total CONS isolates and Methicillin resistance Staphylococcus aureus (MRSA) in $50 \%$ of total Staphylococcus aureus isolates.

Among Gram negative isolates (74), the predominant isolate was Acinetobacter spp $25(33.78 \%)$ followed by Klebsiella spp 22(29.72\%), Citrobacter freundii 9 (12.16\%), Escherichia coli 5 (6.75\%), Pseudomonas aeruginosa 5(6.25\%), NFGNB 4 (5.4\%), Enterobacter aerogenes 3(4\%) and Salmonella typhi 1 (1.35\%) (Table 3).

Antibiotic sensitivity pattern of Gram positive and Gram negative organisms was studied. CONS was $100 \%$ sensitive to Vancomycin and Linezolid, followed by Clindamycin (61.12\%), Tetracycline (56.14\%), Amikacin and Gentamicin $(47.76 \%$ each).
Staphylococcus aureus was $100 \%$ sensitive to Vancomycin and Linezolid, followed by Clindamycin $(62.5 \%)$ and Ciprofloxacin, Oflaxacin, Levofloxacin, Amikacin, Gentamicin and Tetracycline (50\% each) respectively. Enterococcus spp showed 100\% sensitive to Vancomycin and Linezolid, followed by 50\% Amoxy-Clav and Penicillin (Table 4).

Out of total 114 CONS isolates, 94 were MRCONS and among 8 Staphylococcus aureus isolates, 4 were MRSA and the incidence of MRCONS and MRSA being $82.45 \%$ and $50 \%$ respectively.

The most effective antibiotic against Gram positive organisms were Vancomycin and Linezolid (100\% each) followed by Clindamycin (60.12\%) and Tetracycline (56.14\%).

The most effective antibiotic against Acinetobacter spp was Colistin (100\%), followed by Imipenem and Meropenem (68.96\% each), Oflaxacin and Levofloxacin (65.50\% each), Ciprofloxacin (58.62\%), Amikacin and Pipercillin-Tazobactam (51.72\% each). Klebsiella spp showed $100 \%$ sensitive to Colistin, followed by $81.8 \%$ to Imipenem and Meropenem. Citrobacter spp was $100 \%$ sensitive to Colistin, followed by $88.88 \%$ to Imipenem and Meropenem, $77.77 \%$ to Amikacin and Oflaxacin, $55.55 \%$ to Pipercillin-Tazobactam, Ciprofloxacin and Levofloxacin. Escherichia coli showed 100\% sensitive to Colistin, followed by Imipenem and Meropenem (80\% each), Amikacin (60\%) (Table 5).

MDR was found in $60.80 \%$ of Gram negative isolates and ESBL were 20.27\%. MDR was found high among Enterobacteriaceae. Carbapenem resistance was seen more among Nonfermenters (31\%) as compared to Enterobacteriaceae (20.2\%) 
The most effective antibiotic against Gram negative organisms were Colistin followed by Imipenem, Meropenem, Amikacin and Oflaxacin.

In the present study, majority of the isolates showed high resistance to commonly used antibiotics belongs to Penicillins (Ampicillin \&Amoxy-Clav), Cephalosporins (Cefotaxime, Ceftriaxone and Ceftazidime), Fluoroquinolones (Ciprofloxacin,Ofloxacin and Levofloxacin) and Aminoglycosides (Gentamicin and Amikacin).

Blood stream infections constitute one of the most serious conditions and associated with high morbidity and mortality as a result, timely detection, identification and antimicrobial susceptibility testing of blood stream pathogens are important.

The gold standard for diagnosis of BSIs is blood culture. ${ }^{[7]}$ The present study gives information about pathogens causing blood stream infections. It also provides information about antibiotic sensitivity pattern that plays an important role in management of septicaemia cases.

In this study, total 1332 blood samples were received, out of which 204 (15.3\%) samples showed growth and $1128(84.68 \%)$ showed no growth, with culture positivity being $15.3 \%$.

Fig.1 Age distribution of patients

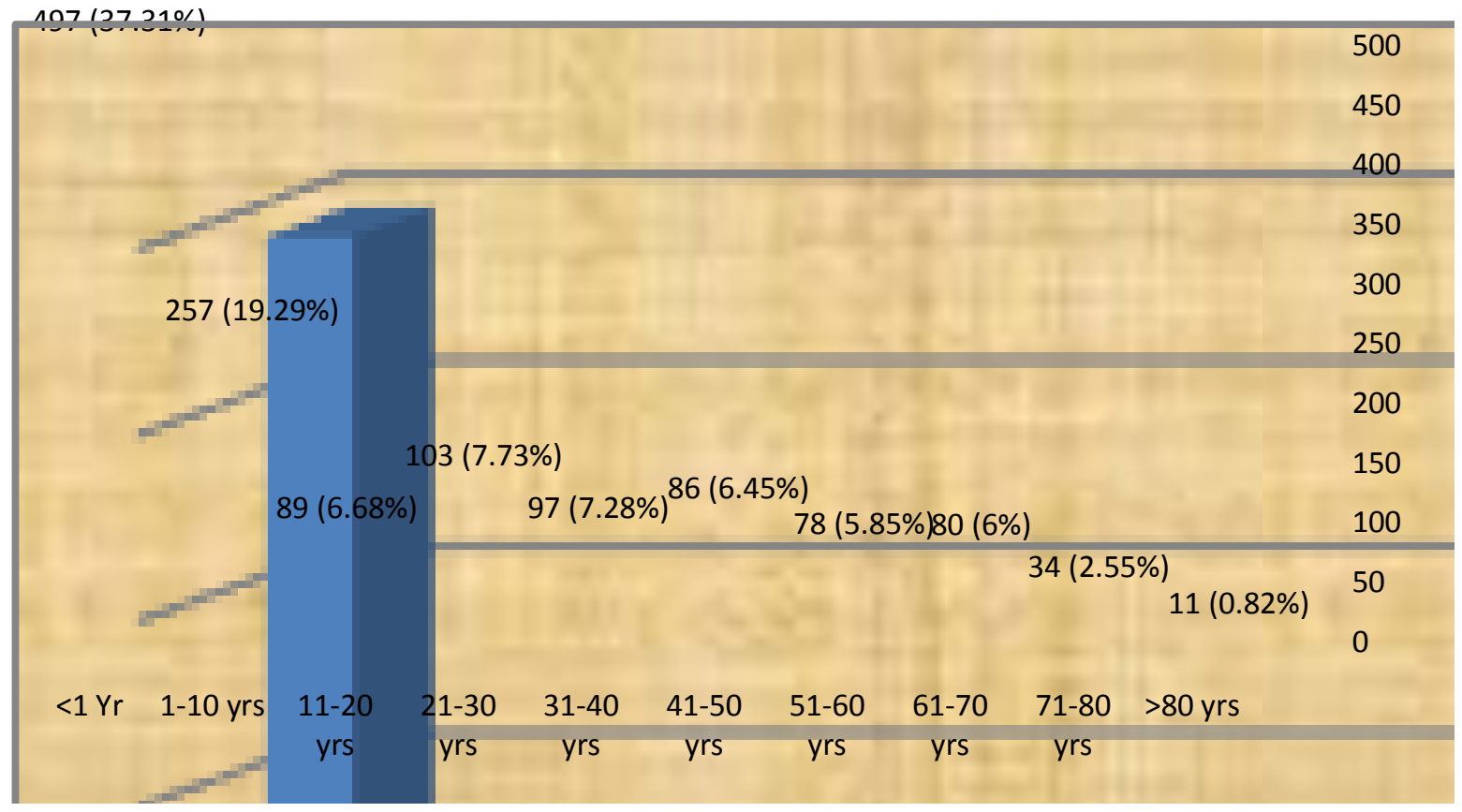

Table.1 Pathogens isolated from samples

\begin{tabular}{|l|c|c|}
\hline Isolates & No. of isolates & Percentage \\
\hline Gram positive bacteria & 128 & $62.74 \%$ \\
\hline Gram negative bacteria & 74 & $36.27 \%$ \\
\hline Candida spp & 2 & $0.98 \%$ \\
\hline \multicolumn{1}{|c|}{ Total } & $\mathbf{2 0 4}$ & $\mathbf{1 0 0 \%}$ \\
\hline
\end{tabular}


Table. 2 Gram positive bacteria isolated from the samples

\begin{tabular}{|c|c|c|c|}
\hline Isolates & & No. of isolates & Percentage \\
\hline $\begin{array}{l}\text { Coagulase negative } \\
\text { (CONS) }\end{array}$ & Staphylococci & 114 & $89 \%$ \\
\hline Staphylococcus aureus & & 8 & $6.25 \%$ \\
\hline Enterococcus faecalis & & 6 & $4.68 \%$ \\
\hline Tota & & 128 & $100 \%$ \\
\hline
\end{tabular}

Table.3 Gram negative bacteria isolated from the samples

\begin{tabular}{|l|c|c|}
\hline Isolates & No. of isolates & Percentage \\
\hline Acinetobacter spp & 25 & $33.78 \%$ \\
\hline Klebsiella spp & 22 & $29.72 \%$ \\
\hline Citrobacter freundii & 9 & $12.16 \%$ \\
\hline Escherichia coli & 5 & $6.75 \%$ \\
\hline Pseudomonas aeruginosa & 5 & $6.75 \%$ \\
\hline NFGNB & 4 & $5.41 \%$ \\
\hline Enterobacter aerogenes & 3 & $4 \%$ \\
\hline Salmonella typhi & 1 & $1.35 \%$ \\
\hline \multicolumn{1}{|r|}{ Total } & $\mathbf{7 4}$ & $\mathbf{1 0 0 \%}$ \\
\hline
\end{tabular}

Table.4 Antibiotic sensitivity pattern of Gram positive isolates

\begin{tabular}{|l|c|c|c|}
\hline & $\begin{array}{c}\text { CONS } \\
\mathbf{n = 1 1 4}(\boldsymbol{\%})\end{array}$ & $\begin{array}{c}\text { Staph.aureus } \\
\mathbf{n = 8 ( \% )}\end{array}$ & $\begin{array}{c}\text { Enterococci spp } \\
\mathbf{n = 6 ( \% )}\end{array}$ \\
\hline Vancomycin & $100 \%$ & $100 \%$ & $100 \%$ \\
\hline Linezolid & $100 \%$ & $100 \%$ & $100 \%$ \\
\hline Clindamycin & $61.12 \%$ & $62.50 \%$ & - \\
\hline Tetracyclin & $56.14 \%$ & $50 \%$ & - \\
\hline Gentamicin & $47.36 \%$ & $50 \%$ & - \\
\hline Amikacin & $47.36 \%$ & $50 \%$ & - \\
\hline Ciprofloxacin & $43 \%$ & $50 \%$ & $16.66 \%$ \\
\hline Oflaxacin & $43 \%$ & $50 \%$ & $16.66 \%$ \\
\hline Levofloxacin & $43 \%$ & $50 \%$ & $16.66 \%$ \\
\hline Erythromycin & $21 \%$ & $37.50 \%$ & - \\
\hline Cotrimoxazole & $14 \%$ & $12.50 \%$ & - \\
\hline Chloramphenicol & $13.15 \%$ & $25 \%$ & - \\
\hline Cefotaxime & $7.90 \%$ & $37.50 \%$ & - \\
\hline Amoxy-Clav & $5.26 \%$ & $12.50 \%$ & $50 \%$ \\
\hline Penicillin & $0 \%$ & $0 \%$ & $50 \%$ \\
\hline
\end{tabular}


Table.5 Antibiotic sensitivity pattern of Gram negative isolates

\begin{tabular}{|c|c|c|c|c|c|c|c|}
\hline & $\begin{array}{c}\text { Acinetobacter } \\
\text { spp } \\
\mathbf{n}=\mathbf{2 5}(\%)\end{array}$ & $\begin{array}{c}\text { Klebsiella } \\
\text { spp } \\
\mathbf{n}=22(\%)\end{array}$ & $\begin{array}{c}\text { Citrobacter } \\
\text { spp } \\
\mathbf{n}=9(\%)\end{array}$ & $\begin{array}{l}\text { E. coli } \\
\mathrm{n}=5(\%)\end{array}$ & $\begin{array}{c}\text { Pseudomonas } \\
\text { aeruginosa } \\
\mathrm{n}=5(\%)\end{array}$ & $\begin{array}{l}\text { NFGNB } \\
n=4(\%)\end{array}$ & $\begin{array}{c}\text { Enterobacter } \\
\text { aerogenes } \\
\mathbf{n}=\mathbf{3}(\%)\end{array}$ \\
\hline Colistin & $100 \%$ & $100 \%$ & $100 \%$ & $100 \%$ & $100 \%$ & $100 \%$ & $100 \%$ \\
\hline Imipenem & $68.96 \%$ & $81.80 \%$ & $88.68 \%$ & $80 \%$ & $80 \%$ & $75 \%$ & $66.66 \%$ \\
\hline Meropenem & $68.96 \%$ & $81.80 \%$ & $88.68 \%$ & $80 \%$ & $80 \%$ & $75 \%$ & $66.66 \%$ \\
\hline Ofloxacin & $65.50 \%$ & $27.27 \%$ & $77.77 \%$ & $0 \%$ & $40 \%$ & $50 \%$ & $0 \%$ \\
\hline Levofloxacin & $65.50 \%$ & $27.27 \%$ & $55.55 \%$ & $20 \%$ & $60 \%$ & $50 \%$ & $0 \%$ \\
\hline Ciprofloxacin & $58.62 \%$ & $22.72 \%$ & $55.55 \%$ & $0 \%$ & $60 \%$ & $50 \%$ & $0 \%$ \\
\hline Amikacin & $51.72 \%$ & $22.72 \%$ & $77.77 \%$ & $60 \%$ & $80 \%$ & $75 \%$ & $0 \%$ \\
\hline $\begin{array}{l}\text { Pipercillin- } \\
\text { Tazobactam }\end{array}$ & $51.72 \%$ & $22.72 \%$ & $55.55 \%$ & $40 \%$ & $60 \%$ & $75 \%$ & $66.66 \%$ \\
\hline Gentamicin & $44.82 \%$ & $22.72 \%$ & $44.44 \%$ & $40 \%$ & $80 \%$ & $75 \%$ & $0 \%$ \\
\hline Tobramycin & $31 \%$ & $13.63 \%$ & $22.22 \%$ & $0 \%$ & $40 \%$ & $50 \%$ & $0 \%$ \\
\hline Amoxy-Clav & $24.13 \%$ & $20 \%$ & $22.22 \%$ & $0 \%$ & $20 \%$ & $25 \%$ & $0 \%$ \\
\hline Cefipime & $24.13 \%$ & $9 \%$ & $33.33 \%$ & $0 \%$ & $40 \%$ & $50 \%$ & $0 \%$ \\
\hline Cefotaxime & $17.24 \%$ & $4.54 \%$ & $22.22 \%$ & $0 \%$ & $0 \%$ & $25 \%$ & $0 \%$ \\
\hline Ceftriaxone & $20.68 \%$ & $4.54 \%$ & $11.11 \%$ & $0 \%$ & $0 \%$ & $25 \%$ & $0 \%$ \\
\hline Ceftazidime & $10.34 \%$ & $4.54 \%$ & $22.22 \%$ & $0 \%$ & $50 \%$ & $25 \%$ & $0 \%$ \\
\hline Ampicillin & $6.89 \%$ & 0 & $5 \%$ & $0 \%$ & $0 \%$ & $0 \%$ & $0 \%$ \\
\hline
\end{tabular}

Blood culture positivity in our study is in comparison with the studies conducted by Pragnya Paramita Jena et al., ${ }^{[10]}(16.2 \%)$, Roy et al.., ${ }^{[1]}$ (16.4\%), Shilpi Gupta et al., ${ }^{[12]}$ (16.5\%), Banik A et al.. ${ }^{[13]}(14.24 \%)$ and China D et al. ${ }^{[14]}(13.9 \%)$. In the study of Sultana et al., ${ }^{[15]}$ and Sharma et al., ${ }^{[16]}$ culture positivity rate was $49.28 \%$ and $33.9 \%$ respectively, which is higher to our study. In contrast low blood culture positivity of $9.9 \%$ by Mehta et al., ${ }^{[17]}, 7.9 \%$ by Anbumanni et al., ${ }^{[18]}, 5.17 \%$ by Barati et al., ${ }^{[19]}$ and $5.6 \%$ by Mehdinejad et al., ${ }^{[20]}$ were reported in similar other studies. The variation in the positivity rate among studies may be due to most of the patient are given antibiotics before they come to the hospital or may be due to selfmedication which is more common. [21] Difference in positivity rate from place to place is also due to different blood culture systems used in laboratories, amount and number of blood culture taken, the study design, and difference in the infection control policies between countries ${ }^{[21]}$

In the present study, among 204 positive cultures, 202(99\%) showed bacterial growth and $2(0.98 \%)$ were Candida spp. This is in comparison with studies conducted by Pragnya Paramita Jena et al. ${ }^{[10]}(96.85 \%$ \& $3.14 \%$ ), Banik et al. ${ }^{[13]}$ (96.66\% and 3.33\%), Qazi et al., ${ }^{[22]}$ (98.4\% and $\left.1.5 \%\right)$ and Shilpi Gupta et al., ${ }^{[12]}$ (96.68\% and 3.31\%).

In the present study, bacteremia due to Grampositive pathogens was more common compared to Gram-negative pathogens. Out of 202 (99\%) bacterial growth, Gram positive organisms were $128(62.74 \%)$ and Gram negative organisms were $74(36.27 \%)$. The predominance of Gram positive bacteria in BSIs was also reported by Banik et al., ${ }^{[13]}$ (GPB 60.37\% and GNB 36.29\%), Pavani et al., ${ }^{[23]}$ (GPB 61.7\% and GNB 38.3\%), Khaleel 
et al., ${ }^{[24]}$ (GPB59.85\% and GNB40.15\%)' Gohel et al., ${ }^{[25]}$ (GPB 58.3\% and GNB 40.2\%), Bhavna Bhadauria et al., ${ }^{[26]}$ (GPB 57.28\% and GNB 42.74\%), Ashima Katayi et al., ${ }^{[27]}$ (GPB 57.14\% and GNB 42.85\%), Dagnew et al., ${ }^{[28]}$ (GPB69\% and GNB31\%) and Wasihun et al., ${ }^{[29]}$ (GPB $72.2 \%$ and GNB $27.8 \%$ ) respectively.

In the present study, among Gram positive isolates (128), the most predominant isolate was CONS 114(89\%) followed by Staphylococcus aureus $8(6.25 \%)$ and Enterococcus spp 6(4.68\%). This finding is in accordance with studies conducted by Karlowsky et al., ${ }^{[30]}$ (CONS 42\%, Staph. aureus $16.2 \%$ and Enterococcus spp 8.3\%), Ashima Katayi et al., ${ }^{[27]}$ (CONS 55.5\%, Staph.aureus $34 \%$ and Enterococcus spp 10.4\%), Alam et al., ${ }^{[31]}$ (CONS 63.5\%, Staph. aureus $23.1 \%$ and Enterococcus spp 5.8\%), Nazir et al., ${ }^{[32]}$ (CONS 67.9\%, Staph. aureus 24.5\%\& Enterococcus spp 7.5\%), Pragnya Paramita Jena et al., ${ }^{[10]}$ (CONS 40.5\%, Staph.aureus $7.87 \%$ and Enterococcus spp $3.1 \%$ ) where CONS reported as the most common isolate causing BSIs.

CONS were mainly recognized as mere contaminants till 1970's; however, several studies have now reported an increasing incidence of infection by this group of bacteria. ${ }^{[32]}$ Over the past two decades, CONS, the usual skin commensals are increasingly being considered blood stream pathogens in select settings. Coagulase negative Staphylococcus is the third most common cause of BSI and the most common cause of nosocomial BSI. ${ }^{[33]}$ Incidence of nosocomial bacteremia due to CONS is increasing due to frequent use of vascular access devices. Improper methods of blood collection and the presence of long standing intravascular catheters are recognized as possible modes of spread of BSI by CONS. 13] Some authors have demonstrated that coagulase-negative Staphylococcus adheres to the catheter surface, and produces slime, which are risk factors for BSI. ${ }^{[34]}$ According to Souvenir et al., clinical significance of CONS was defined as at least two blood cultures positive for CONS within 5 days or one positive blood culture plus clinical evidence of infection, which includes abnormal leukocyte count and temperature or blood pressure. ${ }^{[35]}$

In this study, among Gram negative isolates (74), Acinetobacter spp was the most predominant organism isolated $(39.18 \%)$, followed by Klebsiella spp (29.72\%), Citrobacter spp (12.16\%), E.coli (6.75\%), Pseudomonas aeruginosa (6.25\%), Enterobacter spp (4\%) and S.typhi (1.35\%). These findings are consistent with other studies conducted by Pragnya Paramita Jena et al. ${ }^{[10]}$, Banik et al. ${ }^{[13]}$, Ashima Katayi et al. ${ }^{[27]}$ and Nazir et al., ${ }^{[32]}$ where Acinetobacter spp and Klebsiella spp have been found to be predominant isolates among Gram negative organisms.

The reason for high rate of isolation of Acinetobacter spp among Gram-negative bacteria may be because of acquisition of infection during hospital stay, as it is one of the commonest pathogen seen in nosocomial infections. Also, their ubiquitous nature in the hospital environment and inadequate infection control practice has continuously raised the incidence of Acinetobacter infections over the past two decades. ${ }^{[36]}$

Apart from Gram positive and Gram negative organisms, Candida albicans were isolated in two positive blood cultures (0.98\%). Similar observation was made by Qazi et al., ${ }^{[22]}$.

The results of antibiotic sensitivity of Grampositive bacteria showed CONS, Staphylococcus aureus and Enterococcus spp were $100 \%$ sensitive to Vancomycin and 
Linezolid followed by Clindamycin and Tetracycline and were least sensitive to Penicillins, Cephalosporins and Fluoroquinolones and this finding similar to other studies. The incidence of MRCONS was $82.45 \%$ and MRSA was 50\%. Methicillin resistance rate was higher in CONS as compared with Staphylococcus aureus, which is similar to study by Mathur et al. ${ }^{[37]}$ and Mir et al., ${ }^{[38]}$.

These organisms are notorious since they do not respond to the broad class of beta lactam antibiotics and acquire resistance to newer antibiotics quite rapidly. This effectively complicates the management of such BSIs. ${ }^{[10]}$ In the present, the most effective antibiotic against Gram positive organisms were Vancomycin and Linezolid followed by Clindamycin and Tetracycline.

Among Gram negative bacteria, Acinetobacter spp, Klebsiella spp, Citrobacter spp, E. coli and NFGNB showed $100 \%$ sensitive to Colistin followed by Imipenem and Meropenem (80\% each), Oflaxacin and Levofloxacin (60\% each) and Amikacin (55\%) and least sensitive to Ampicillin, Amoxicillin+clavulanic acid combination and Cephalosporins and this is similar to other studies.

In our study MDR was found in $60.80 \%$ of Gram negative isolates and is in comparison with study conducted by Shilpi Gupta et al., ${ }^{[12]}$ and Nazir et al., ${ }^{[32]}$ and ESBL were $20.27 \%$ which is similar to study conducted by Anathan et al., ${ }^{[39]}$ (25.4\%). MDR was found high among Enterobacteriaceae. Carbapenem resistance was seen more among Nonfermenters $(31 \%)$ as compared to Enterobacteriaceae $(20.2 \%)$ and this may be due to inappropriate empirical use of Carbapenem as the first line treatment.

The greatest threat with MDR and Carbapenem resistant Gram negative bacteria is that the infections are usually untreatable due to the limited options of the antibiotics available, resulting into increased mortality. Worldwide, their incidence is rising with variations due to regional and geographical differences as stated by Jadhav et al., ${ }^{, 40]}$. With the shortage of newer drugs availability and increasing resistance, use of limited option drugs such as colistin by clinicians could soon lead to the condition of so called pan drug resistance. ${ }^{[12]}$ In the present study, the most effective antibiotic against Gram negative organisms were Colistin followed by Imipenem, Meropenem, Amikacin and Oflaxacin.

The information of predominant organisms and their sensitivity among sepsis patients is essential for making the right choice of antibiotics in the management of sepsis. Hence, blood cultures must be obtained from all suspected cases of bacteraemia or sepsis before prescribing antibiotics. The main factors causing the increase in antimicrobial resistant bacteria are poor infection control practices and inappropriate use of antibiotics. Strict infection control measures along with antibiotic policy for judicious antibiotic therapy should be implemented in the hospitals as control measures against blood stream infections and to check the emergence of resistance. ${ }^{[41]}$

Blood stream infections are an important nosocomial infection responsible for morbidity and mortality in the patients. The present study provides information on the spectrum of pathogens causing blood stream infections and their antimicrobial susceptibility profile, helping the clinicians in early diagnosis and guiding in the management of blood stream infections. The study identified both Gram-positive and Gram-negative bacteria to be responsible for blood stream infections and most of them were found to be MDR. Inappropriate antibiotic use and poor infection control 
practices contributes to the emergence of antimicrobial resistance in bacteria. The key to control of antibiotic resistant pathogens is to strictly adhere to infection control practices and mandates antibiotic policy for rational use of antibiotics. Also, Routine surveillance of antimicrobial resistance in frequently encountered bacterial pathogens will be useful for deciding on empirical treatment strategies and also devising an effective antimicrobial stewardship program in hospitals.

\section{References}

1. Meremkwer MM, Nwachukwu CE, Asuquo AE, Okebe J, Utsalo SJ. Bacterial isolates from blood cultures of children with suspected septicaemia in Calabar, Nigeria. BMC Infect Dis. 2005; 5:110-5.

2. Claudio Viscoli Virulence. 2016 Apr; 7(3): 248-251.

3. Healthcare Related Infection Surveillance and Prevention (CHRISP) Signal Infection Surveillance Manual, Section 3 Blood Stream Infection Signal. Jun 2013.

4. CDC/NHSN Surveillance HAI Criteria. Jun 2013.

5. Seifert $\mathrm{H}$, Wisplinghoff $\mathrm{H}$. Bloodstream infection and endocarditis. In: Borriello SP, Murray PR, Funke G, editors. Topley and Wilson's Microbiology and Microbial Infections, Bacteriology. 10th ed., Vol. 1. Ch. 4.1. London: Hodder Arnold ASM Press; 2005. p. 1181- 235.

6. Basic laboratory procedures in clinical bacteriology. $2^{\text {nd }}$ edition. World Health Organization, Geneva. 2004; pp. 20-21.

7. Wadud ABMA. Bacteriological profiles of blood culture isolates by BacT/ALERT 3D automated system. Journal of Shaheed Suhrawardy Medical College. 2009; 1(2): 213-219.
8. French GL. Clinical impact and relevance of antibiotic resistance. Adv Drug Deliv Rev. 2005; 57(10): 1514-27.

9. Clinical and Laboratory Standards Institute. Performance Standards for Antimicrobial Susceptibility Testing: Twenty- Fourth Informational Supplement. CLSI Document M100- S27. Wayne, PA: Clinical and Laboratory Standards Institute; 2017.

10. Pragnya Paramita Jena, Renu Gur, Shalini Dewan Duggal.Microbiological Profile and Antibiogram of Blood Stream Isolates at a Referral Hospital in North Delhi: A One Year Study. International Journal of Biomedical Research 2015; 6(10): 819-824.

11. Roy I, Jain A, Kumar M, Agarwal SK. Bacteriology of neonatal septicaemia in a tertiary care hospital of Northern India. Indian J Med Microbiol 2002; 20: 156-159.

12. Shilpi Gupta, Bineeta Kashyap. Bacteriological profile and antibiogram of blood culture isolates from a tertiary care hospital of North India. Trop J Med Res 2016; 19: 94-9.

13. Banik A, Bhat SH, Kumar A, Palit A, Snehaa K. Blood stream infections and trends of antimicrobial sensitivity patterns at Port Blair. J Lab Physicians 2018; 10: 332-7.

14. Chinna D et al., Bacterial profile and antimicrobial susceptibility pattern of blood isolates from a tertiary care hospital in North India. IJPRBS 2013; 2(2): 24-35.

15. Sultana Q, Ansari H, Ansari WMA. Bacteriological profile and antimicrobial susceptibility patterns of organisms responsible for blood stream infections. Indian J Microbiol Res 2016; 3(2): 1137.

16. Sharma M, Goel N, Chaudhary U, Aggarwal R, Arora DR. Bacteraemia in 
children. Indian J Pediatr 2002; 69: 1029: 32.

17. Mehta M. et al., antimicrobial susceptibility pattern of blood isolates from a teaching hospital in north India. Jap J Infect Dis 2005; 58: 174-76.

18. Anbumani N. et al., Distribution and Antimicrobial Susceptibility of Bacteria Isolated from Blood Cultures of Hospitalized Patients in a Tertiary Care Hospital. Indian journal of practicing doctor 2008; 5(2): 75-79.

19. Barati M. et al., Bacterial profile and antimicrobial susceptibility of blood culture isolates; Iran J. Med Sci 2009; 4(2): 87-95.

20. Mehdinejad M. et al., Study of prevalence and antimicrobial susceptibility pattern of bacteria isolated from blood cultures. J. Biologic Sci 2009; 9(3): 249-53.

21. Lee A, Mirrett S, Reller LB, Weinstein MP. Detection of blood stream infections in adults: How many blood cultures are needed? J ClinMicrobiol 2007; 45: 3546- 8 .

22. Dr M.S. Qazi, Dr Bhawana Bajare, Dr Neha Baid. Study of microbial profile and antibiogram of blood stream infections in adults with septicemia. Indian journal of research 2017; 6 (12): 414-416.

23. Pavani, G., et al., Bacterial pathogens responsible for blood stream infection and pattern of drug resistance in a tertiary care hospital of Lahore. Biomedica 2009; 25 (3): 142-149.

24. Khaleel M.E. et al., Study of Microbial Isolates from Blood at a University Teaching Hospital Annals 2010; 16(3): 25-29.

25. Gohel, K. et al., Bacteriological Profile and Drug Resistance Patterns of Blood Culture Isolates in a Tertiary Care Nephrourology Teaching Institute.
BioMed Research International 2014: 15.

26. Bhadauria B, Farooq U, Singh S, Dayal N, Mashkoor S, Sridhar D. Bacteriological Profile and Antibiogram of Gram Negative Bacteria Isolated from Blood Culture. Int Arch BioMed Clin Res 2017; 3(2): 91-95.

27. Ashima Katayi, Deepinder Singh, Madhu Sharma, Uma Chaudhary. Bacteriological profile and antibiogram of blood culture isolates from intensive care units in a teaching tertiary care hospital. Journal of Health Sciences and Reasearch 2018; 9(1): 6-10.

28. Dagnew M, Yismaw G, Gizachew M, Gadisa A, Abebe T, Tadesse T, Alemu A. Bacterial profile and antimicrobial susceptibility pattern in septicemia suspected patients attending Gonder University Hospital, Nortwest Ethiopia.BMC Res Notes 2013; 6: 283.

29. Wasihun AG, Wlekidan LN, Geremariam SA, Dejene TA, Welderufael AL, Haile TD, Muthupandian S. Bacteriological profile and antimicrobial susceptibility pattern of blood culture isolates among febrile patients in Mekelle Hospital, Northern Ethiopia. Springer plus 2015; 4: 314.

30. Karlowsky JA, Jones ME, Draghi DC, Thornsberry C, Sahm DF, Volturo GA, et al., Prevalence and antimicrobial susceptibilities of bacteria isolated from blood cultures of hospitalized patients in the United States in 2002. Ann Clin Microbiol Antimicrob 2004; 3: 7.

31. Alam MS, Pilai PK, Kapur P, Pillai KK.Resistant pattern of bacteria isolated from blood stream infections at a university hospital in Delhi. J Pharm Bioallied Sci 2011; 3(4): 525-530.

32. Asifa Nazir, Ifshana Sana, Bushra Yousuf Peerzada, Tabindah Farooq. Study of prevalence and antimicrobial susceptibility pattern of blood culture 
isolates from a tertiary care hospital of North India. Int J Res Med Sci 2018; 6(12): 4046-4052.

33. Pfaller MA, Jones RN, Doern GV, Sader HS, Kugler KC. Survey of blood stream infections attributable to grampositive cocci: frequency of occurrence and antimicrobial susceptibility of isolates collected in 1997 in the United States, Canada and Latin America from the SENTRY Antimicrobial Surveillance Program, SENTRY Participants Group. Diagn Microbiol Infect Dis 1999; 33: 283-297.

34. Raad, I., et al., "Serious complications of vascular catheter-related Staphylococcus aureus bacteremia in cancer patients." European Journal of Clinical Microbiology and Infectious Diseases Vol 1992; 11(8): 675-682.

35. Souvenir D, Donald E, Anderson J, Palpant S, Mroch H, Askin S, Anderson J, Claridge J, Eiland J, Malone C, Garrison MW, Watson P, Campbell DM. Blood Cultures Positive for Coagulase-Negative Staphylococci: Antisepsis, Pseudobacteremia, and Therapy of Patients. J Clin Microbiol 1998; 36(7): 1923-1926.

36. Prashanth K, Badrinath S. Nosocomial infections due to Acinetobacter species: Clinical findings, risk and prognostic factors. Indian J Med Microbiol 2006; 24: $39-44$
37. Mathur P, Varghese P, Tak V, Gunjiyal J, Lalwani S, Kumar S, Misra MC. Epidemiology of blood stream infections at a level-1 trauma care center of India. J Lab Physicians 2014; 6(1): 22-27.

38. Mir AB, Srikant. Prevalence and antimicrobial susceptibility of methicillin resistant Staphylococcus aureus and coagulase negative Staphylococci in a tertiary care hospital. Asian J Pharm Clin Res 2013; 6(3): 231-134.

39. Ananthan S, Subha A. Cefoxitin resistance mediated by loss pf a porin in clinical strains of Klebsiella pneumoniae and E.coli. Indian J Med Microbiol 2005; 23(1): 20-23

40. Jadhav S, Gandham N, Paul R, Misra RN, Ujagare MT, Angadi K. Bacteriological profile of septicaemia and antimicrobial susceptibility of isolates from tertiary care hospital in India. Res J Pharm Biol Chem Sci 2012; 3: 1100- 8 .

41. Vijay Prakash Singha, Abhishek Mehta. Bacteriological profile of blood stream infections at a Rural tertiary care teaching hospital of Western Uttar Pradesh. Indian Journal of Basic and Applied Medical Research 2017; 6(3): 393-401.

\section{How to cite this article:}

Vidyasagar, K. and Venkatesha, D. 2019. Study of Microbiological Profile and Antibiotic Susceptibility of Blood Stream Infections in Tertiary Care Hospital. Int.J.Curr.Microbiol.App.Sci. 8(07): 1201-1211. doi: https://doi.org/10.20546/ijcmas.2019.807.143 